\title{
Opening the radio sky to all
}

\author{
André Gilloire \\ Association Observation radio - Pleumeur-Bodou \\ ABRET-COSMOPOLIS, 22560 Pleumeur-Bodou, France \\ email: andre.gilloire@wanadoo.fr
}

\begin{abstract}
The purpose of our project is to convert a large $13 \mathrm{~m}$ parabolic antenna formerly used for satellite communications in the decimetric range into a radio telescope dedicated to pedagogy and scientific culture spreading. This project has been labeled within the framework of AMA 2009 with other similar projects in France. The paper explains the project and gives details about its collaborations and partnerships.
\end{abstract}

Keywords. Radio astronomy, HI observation, RFI mitigation, pedagogy, outreach

\section{Introduction}

Radio astronomy is much less known by the public than "traditional" optical astronomy, even though it lies at the root of major discoveries (cosmological microwave background, pulsars, radio galaxies, study of galaxy dynamics through Hi radio emission, etc) and in spite of its increasing importance for the knowledge of the universe. The availability of a large parabolic antenna formerly used for satellite communications opened an opportunity to start a high range project with pedagogic purposes. The antenna is installed in the historical site of Pleumeur-Bodou, in North Brittany, France, where the first TV satellite transmission between USA and Europe took place in 1962. The site still hosts 3 large parabolic antennas, relics of one of the largest satellite communication centers in the world, which was active un to the year 2000. It is quiet and attractive for radio observation of the sky. The smallest $(13 \mathrm{~m}$ ) of these antennas, named PB8 (Figure 1), is still in good condition and is fairly easy to convert and upgrade for a new use as a radio telescope. The site also contains the Cité des Télécoms, a science and technology museum dedicated to telecommunications history and current releases, and is close to the Planétarium de Bretagne, hence two leading structures in Brittany for outreach on science and technology. The Association Observation radio - Pleumeur-Bodou, launched in 2007, seized this opportunity to start and develop the project, with the help of other local associations (APAST , ABRET ) involved in scientific outreach and pedagogy. The project is carried out by amateurs enthusiastic about astronomy and radio technology and is financially supported through contracts.

\section{Project prospects for the International Year of Astronomy and beyond}

The main results in 2009 are: (1) demonstrations of radio observations of our Galaxy (HI radiation at $21 \mathrm{~cm}$ ) and of the Sun; (2) exhibitions and conferences on radio astronomy and cosmology in cooperation with the Planétarium de Bretagne and other structures and associations. Over the next years, the main objectives of the project are: (a) development of the pedagogical activities for the public and for students, summer schools, workshops; (b)to start and develop a research program on RFI mitigation for radio astronomy in cooperation with astronomers, institutions and engineering schools, 
summer school on that topic in 2010; (c) conversion of the largest (30 m) antenna in the site to a radio-telescope compliant with the standards of the VLBI network (blue sky dream, since a large funding is needed for this!)

\section{How the project is organised and supported}

The project is carried out by a staff of 12 members: engineers, professors, scientists, teachers, mainly from Brittany. All members are volunteers and are science and technology enthusiasts. It is organised in several working groups: antenna mechanics and steering system, radio equipment, communication and outreach, project management. Indeed, most members belong to several of theses groups. An original feature of the project is that it has a scientific advisory board of 5 members who are scientists and university / engineering schools teachers. Research contracts with institutions such as the French CNES bring the essential financial support. The antenna structure and building are owned by the local administrative authority (Lannion Trégor Agglomération) and are maintained by them.

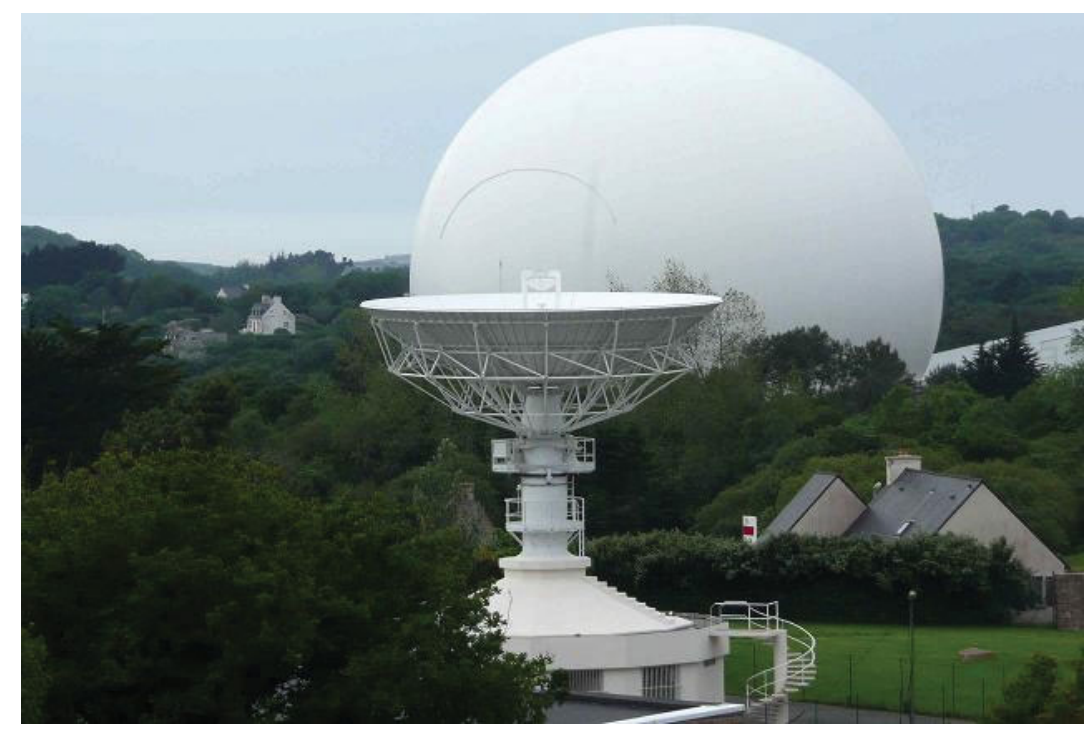

Figure 1. The $13 \mathrm{~m}$ antenna.

The project is taking part in a network of six French observatories contributing to a global project: "Observations of the Galaxy for All", involving radio telescopes dedicated to pedagogy $\dagger$. They will perform observations of our Galaxy in the Hi band around 1420 $\mathrm{MHz}$ and will be open to the public. This global project has been labeled at the French national level within the frame of IYA 2009. Connections are also being considered with the E-HOU project, especially with the SALSA project $\ddagger$ led by the Onsala observatory in Sweden 9 . Development of contacts and cooperations in Europe and throughout the world are one of our objectives. We are also considering the adaptation of the Haystack SRT receiver\| to the $13 \mathrm{~m}$ antenna.

$\dagger$ see http://aramis.obspm.fr/ ${ }^{\sim m e l c h i o r / A M A 09 p u b / r a d i o t e l . h t m ~}$

$\ddagger$ see http://www.chalmers.se/rss/oso-en/observations/2-3-m-lab-antenna-salsa I see http://www.ehou.net for details.

|| see http://www.haystack.mit.edu/edu/undergrad/srt/index.html 